\title{
International Olympic Committee Expert Group Statement on Dietary Supplements in Athletes
}

Dietary supplements encompass a wide range of products, including essential nutrients (vitamins, minerals, proteins, amino acids, etc.), herbals and botanicals, and specific products with potential for maintenance of health and optimisation of performance. The use of dietary supplements is widespread among elite athletes, as it is in the general population. Users cite many different reasons for consuming dietary supplements, though these reasons are often based on unfounded beliefs rather than on any clear understanding of the issues at stake, and may reflect encouragement from individuals who are influential rather than being experts on this topic. There is no universal system to categorise the supplements used by athletes, but it can be helpful to divide them (or their uses) into products that address specific nutrient deficiencies, sports foods, performance supplements, and supplements for health, adaptation or physique change.

There is now widespread acceptance that some of these dietary supplements can offer benefits to the elite athlete if used appropriately, but that some may be harmful to health and/or performance. Benefits from the use of supplements and sports foods may include convenience and provision of a known amount of a key nutrient, as, for example, in the use of protein supplements after training with the aim of promoting traininginduced adaptations in muscle and other tissues. Supplements should be considered only when the risks are low and a health or performance benefit is likely, but there is a limited evidence base for most supplements. There is a clear need for well-conducted and sports-specific research on supplements, as many of the published studies have used inappropriate experimental models and subject populations that are not representative of the elite athlete. Assessment of the evidence requires a consideration of potential limitations to study design, including confounding variables and bias, and of the relevance to real-life practices of elite athletes, as well as the need for verification of the composition of supplements used. Supplement-induced performance changes should be interpreted in light of what is meaningful to the outcome of sporting competition. It must be remembered, though, that absence of evidence of efficacy is not the same as evidence of absence of efficacy.

A comprehensive nutrition assessment is the first step in advising athletes on dietary strategies or medical uses of supplements. Nutrition assessment requires the systematic collection, verification and interpretation of the data needed to identify nutrition-related problems, their causes and their significance. A complete assessment should include dietary evaluation, body composition analysis, biochemical testing, nutrition-focused clinical examination, and patient health and performance history. Assessment should take account of maturation status, sex, ethnicity and culture. The limitations and uncertainties in all of the methods employed must be recognised, though valid and reliable methods are available for some specific nutrients.

Only after an athlete has reached an appropriate level of maturity and competition-readiness, and has a good training, recovery and nutrition plan in place, should the potential of performance supplements to provide further marginal gains be explored. A few specific supplements may offer performance benefits to some athletes, but their use requires careful evaluation. Supplements supported by good evidence of efficacy, in at least some exercise models, include carbohydrate, protein, caffeine, creatine, specific buffering agents and nitrate. Benefits may be mediated by addressing the physiological factors that limit performance in a specific sporting scenario and/or by effects on the central nervous system. Because responses seem to vary between individuals and depend on the exercise model used, supplements should be thoroughly trialled in training or simulated competition before implementation into a competition environment. Indeed, it is possible that deleterious responses may outweigh any expected performance-enhancing affect.

An increasingly-recognised hazard related to supplement use has emerged: inadvertent ingestion of substances that are prohibited under the anti-doping codes that govern elite sport, but are present in some supplement products. In some cases, the level of prohibited or toxic substances in supplements presents a health hazard for all consumers. In other cases, the content may be too small to cause any health or performance effect, but large enough to record an Anti-Doping Rule Violation for athletes who submit to anti-doping tests. These problems may arise from poor quality assurance during production or from deliberate adulteration of otherwise ineffective products.

Practitioners who work with Olympic and highly competitive athletes know that the pressures of elite sport and the substantial rewards that follow success provide a high level of motivation to adopt any safe and legal strategy that might promise even the smallest performance gain. Dietary supplements operate in this space, whether they promise a large performance boost or just create the fear that an athlete cannot afford to miss out on what their rivals are using. A risk-benefit analysis should be undertaken before any decision is made to use a supplement, but many elite athletes mistakenly believe that the rewards of sporting success outweigh the lack of evidence to support the claims made for many supplements. Athletes must appreciate, however, that the consequences of an Anti-Doping Rule Violation are potentially catastrophic. The use of dietary supplements should not compensate for poor food choices and an inadequate diet, except as a short-term strategy when nutrient intake is challenged or dietary changes are not possible. Use of products that have been subjected to one of the available quality assurance schemes can help to reduce, but not eliminate, the risk of an inadvertent doping infringement. Vulnerable populations, including young athletes, may require particular 
support in making informed choices about supplement use. In general, use of supplements by young athletes is discouraged except when full evaluation of nutritional status suggests that it is warranted.

Protection of the athlete's health and awareness of the potential for harm must be paramount. Therefore, expert professional help should be sought before a consensual decision on supplement use is made.

Lausanne, Switzerland, 5 May 2017

\section{Endnote}

This Expert Group Statement presents the conclusions of the International Olympic Committee (IOC) Medical and Scientific Consensus Meeting on Supplements, held May 3-5, 2017, at the IOC headquarters in Lausanne, Switzerland. For more information, including the full list of Expert Group participants, visit https://www.olympic.org/news/ioc-tackles-the-topic-of-nutritionalsupplements-their-use-and-their-effects. 